Document downloaded from:

http://hdl.handle.net/10251/156839

This paper must be cited as:

Morant, M.; Llorente, R. (2019). Performance analysis of multiple radio-access provision in a multicore-fibre optical fronthaul. Optics Communications. 436:161-167.

https://doi.org/10.1016/j.optcom.2018.11.036

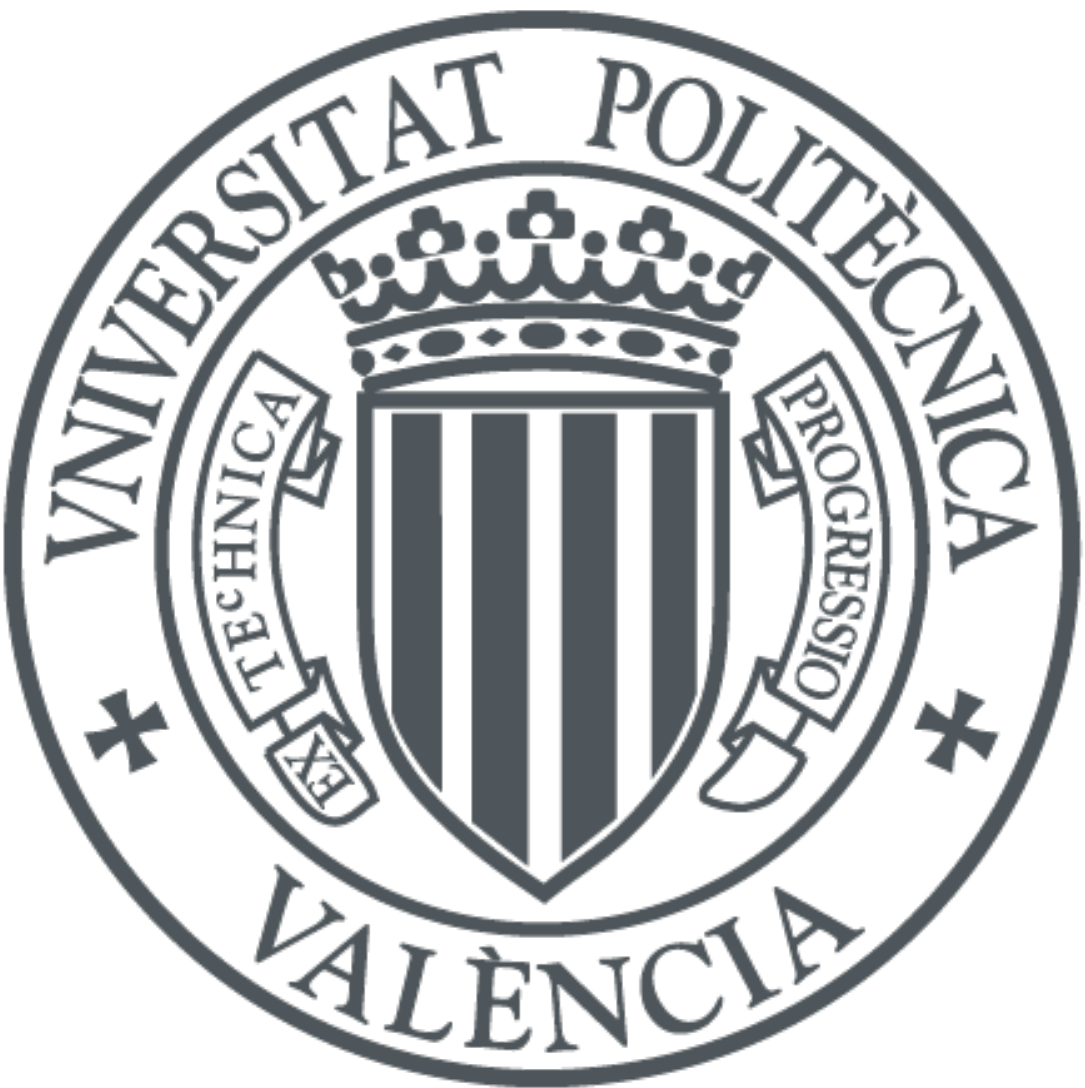

The final publication is available at

https://doi.org/10.1016/j.optcom.2018.11.036

Copyright Elsevier

Additional Information 


\title{
Performance Analysis of Multiple Radio-Access Provision in a Multicore-Fibre Optical Fronthaul
}

\author{
Maria Morant and Roberto Llorente \\ Nanophotonics Technology Center, Universitat Politècnica de València, Spain
}

\begin{abstract}
In this work we report a comprehensive experimental study targeting the dimensioning of the next-generation multicore-fibre (MCF) optical fronthaul employing space-division multiplexing (SDM). This fronthaul is capable of simultaneous provision of multiple radio-access technologies (multi-RATs) with advanced multi-antenna MIMO capabilities per RAT. The different parameters required for fronthaul dimensioning are evaluated considering state-of-the-art 4G LTE-Advanced altogether other legacy wireless standards in operation nowadays. In particular, the modulation characteristics, the antenna quality requirements (in terms of EVM, phase error or rho) and the signal-to-noise ratio (SNR) thresholds are evaluated employing fully-standard cellular signals transmitted on a multicore fibre (MCF) fronthaul. The study includes bi-directional signal transmission and multi-antenna MIMO multiplexing. The MCF optical fronthaul is evaluated with a multiplexed transmission of 2G, 3G, 3.9G and 4G MIMO signals in radio-over-multicore-fibre (RoMCF) employing commercially available four-core MCF. The received SNR requirements are obtained for each cellular signal considering GSM, EDGE, EGPRS2-A, cdma2000 1xEV-DO, UMTS HSPA+ and LTE-Advanced. Single-antenna and two-antenna systems implementing $2 \times 2$ MIMO transmission can be accomplished with SNR levels over $25 \mathrm{~dB}$. In the case of $4 \times 4$ MIMO multiplexing over four cores of MCF fronthaul transmission, $32 \mathrm{~dB}$ SNR is needed to achieve almost four times the provided bitrate per user.
\end{abstract}

Keywords: Optical fronthaul, multi-RAT, multicore fibre, radio-over-fibre, space-division multiplexing, MIMO radio systems

\section{Introduction}

Next-generation radio-access networks are expected to require massive fronthaul capacity when considering support for the fifth-generation (5G) cellular technology [1]. 5G will support different profiles operating at different frequency bands including sub-GHz spectrum bands targeting longrange coverage in rural or remote areas, spectrum bands around $3 \mathrm{GHz}$ to provide connectivity in dense urban areas, and higher frequency bands in the mm-wave range for dedicated highperformance small-cell connectivity (5G NR, new-radio)[2]. This implies that 5G operators are set to deploy cellular networks with a large number of remote radio heads (RRH) on the field, some of them providing joint service of current $(5 \mathrm{G}, 4 \mathrm{G})$ and legacy $(3 \mathrm{G}, 2 \mathrm{G})$ wireless, to optimize the network deployment cost [3]. Compared with $4 \mathrm{G}$ radio access, in order to support a wider range of services and scenarios, 5G will need to be more massive and scalable [4]. Early 5G trials indicate that user provision of massive amounts of data with minimal delay stresses the requirements of conventional fronthaul solutions [4][5]. The capacity requirements are further stressed when $100 \mathrm{MHz}$ channels and massive MIMO technology is considered [6]. Centralized/cloud radio access networks (C-RANs) have been proposed as a convenient solution when a massive number of RRHs must be supported, being also under consideration in early beyond-5G network architecture studies reported [7].

C-RAN requirements for $5 \mathrm{G}$ are highly challenging for CPRI-based fronthaul optics [6]. Analog radio-over-fibre (RoF) transmission has been appointed as a promising technology to provide $5 \mathrm{G}$ network objectives including seamless coverage, gigabit-per-second user capacity and green communications [8]. Although digital-RoF has also been proposed, analog-RoF is less complex, more flexible and transparent, while demanding less bandwidth than digital-RoF [9][10]. Recently, different optical fronthaul technologies have been evaluated for C-RAN implementation by analysing the deployment costs and capabilities to support advanced wireless functionalities and to fulfil the $5 \mathrm{G}$ latency and capacity requirements. This analysis pointed out that analog radio-over-fibre technology not only satisfies the primary $5 \mathrm{G}$ requirements but also reduces the deployment cost 
compared to the traditional C-RAN architecture [1]. By implementing RoF in the fronthaul, expensive analog-to-digital (ADC) and digital-to-analog converters (DAC) are centralized in the site with the digital and radio units, leading to reduced deployment costs [11]. Moreover, analog RoF technology presents better timing, leading to a lower delay compared to CPRI over a few $\mathrm{km}$, in accordance with the expected optical interconnection range between the baseband unit (BBU) and the remote radio head in a 5G cellular network. It also provides better flexibility supporting multiple cellular wireless signals, i.e. 5G radio provision jointly with $4 \mathrm{G}$ and legacy cellular services operating at different frequency bands [1]. The proposed multi-RAT architecture is depicted in Fig. 1, where fronthaul optical transmission is supported by a multicore optical fibre where different services can be allocated to different cores.

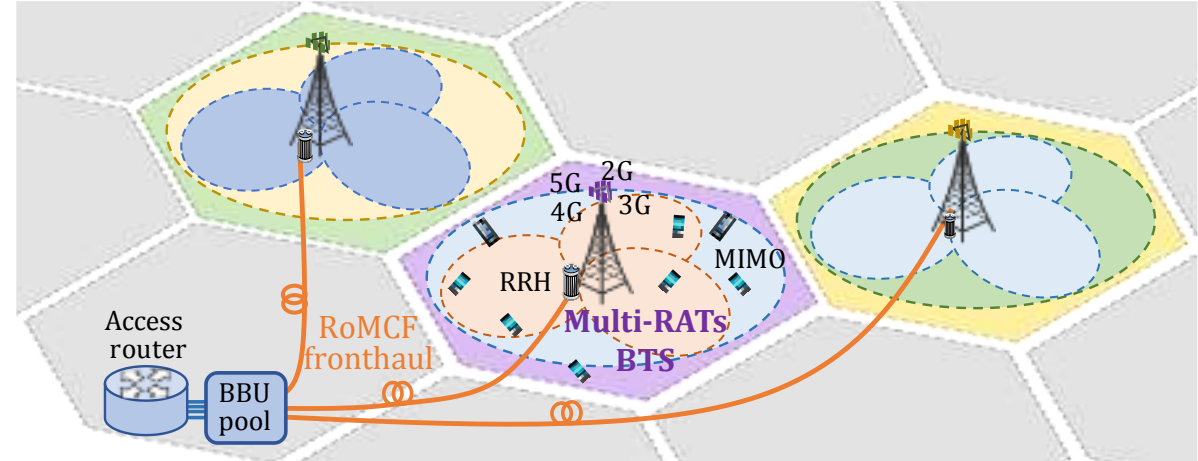

Fig. 1. Conceptual C-RAN architecture supporting multi-RATs and MIMO provision with RoMCF fronthaul

Although the transmission capacity of standard single-mode single-core fibre has increased in the last decades due to advanced transmission technologies, we are approaching to its fundamental limit around $100 \mathrm{~Tb} / \mathrm{s}$ owing to the limitation of fibre fuse, amplifier bandwidth and nonlinear noise [12]. In order to further increase the fibre capacity, space division multiplexing (SDM) over different cores in a multicore fibre (MCF) was firstly proposed in 1979 [13]. Later in 2010, when the capacity crunch became a reality [14], MCF appeared as a strong SDM medium candidate for next generation optical networks [15]. MCF comprises multiple cores with sufficiently low crosstalk between neighbouring cores in a single fibre to provide separated spatial paths [12]. Next-generation Mobile Internet Communication will require unlimited and seamless access to Internet information between different network standards [3]. Next-generation networks need to deal with many more base stations, deployed dynamically in heterogeneous multiple radio access technologies (also known as multi-RATs), such as GSM, UMTS or LTE, which need to be flexibly integrated in the network [16].

Fig. 1 depicts a fronthaul scenario implementing radio-over-multicore-fibre (RoMCF) to provide multi-RATs coexistence and multi-antenna MIMO capability. Future network scenarios for ultradense areas and with multi-RATs coexistence will require the deployment of various fronthaul links of C-RAN for each RAT [16] that could be avoided by using multicore fibre fronthaul links. As it was demonstrated recently, MCF can be used for the reconfigurable radio-over-fibre provision of cellular signals in the fronthaul link [17]. The use of SDM on MCF provides multiple spatial paths for the optical signals corresponding to heterogeneous multi-RATs with the main advantage that for a givenwavelength, the chromatic dispersion is the same for all optical paths. This minimizes the impact of the optical transmission compared with WDM systems. It also enables the spatial multiplexing of different data streams as defined in 3GPP to improve network capacity with multi-antenna MIMO or joint beamforming, where the latency between the signals is of utmost importance [16]. In addition to this, RoMCF transmission of spatial-multiplexed MIMO over different cores of a MCF link takes advantage of the digital signal processing algorithms already in-place in commercially available 3GPP equipment, such as LTE-Advanced (LTE-A) devices [18]. As a novelty of this work, we evaluate experimentally the performance of different cellular signals for the coexistence of multi-RATs in RoMCF transmission considering GSM, EDGE, EGPRS2-A, cdma2000 1xEV-DO, UMTS HSPA+ and LTE- 
Advanced full-standard signals. The experimental study includes the evaluation of bi-directional signal transmission when the modulation characteristics of the downlink (DL) and uplink (UL) signals are different. We also evaluate experimentally the requirements for state-of-the-art 4G multiantenna systems with spatial multiplexing of different data streams over the different cores of the MCF implementing MIMO.

This paper is structured as follows: In Section II, the main characteristics of the fully-standard cellular signals generated for the experimental evaluation are summarized, including the signal quality requirements at the antenna defined by each standard. Next, in Section III, the experimental performance of a RoMCF fronthaul link is evaluated for different cellular services over a four-core fibre. The requirements in terms of received signal to noise ratio (SNR) are evaluated for each service in order to meet the signal quality recommended by each cellular standard. The experimental evaluation includes bi-directional service provision and the evaluation of multi-antenna signals spatially multiplexed over different cores with $2 \times 2$ and $4 \times 4$ MIMO implementations. Finally, in Section IV, the main conclusions of this work are highlighted.

\section{Experimental Setup for Multi-RATs Signal provision over Multicore Fibre}

Fig. 2 and Fig. 3 show the experimental setups developed for the RoMCF transmission of different cellular services through $150 \mathrm{~m}$ of MCF, emulating a fronthaul link supporting multi-RATs. Each cellular service is modulated over a $1555.75 \mathrm{~nm}$ continuous wave (CW) laser employing MachZehnder electro-optical modulators operating at quadrature bias point. When the modulation characteristics of the DL and UL signals are different, we employ the experimental setup depicted in Fig. 3 to evaluate the performance in both directions over different cores of the MCF. The electrical signals for the multi-RATs provision are generated fully compliant with current cellular standards using a wireless test set (Keysight E6640A EXM). The cellular signals are generated at the centre of the regulated frequency band of 2G, 3G, 3.9G and 4G services in Spain. However, the centre frequency of multi-RATs services could be easily changed at the BBU as the electro-optical devices employed in this demonstration have a bandwidth higher than $10 \mathrm{GHz}$ to allocate different frequency bands.

The modulated signals are injected to the 4-core fibre (4CF) Fibrecore SM-4C1500(8.0/125) with a 3D fan-in, injecting $<-1 \mathrm{dBm}$ optical power in each core. The signals are extracted with similar 3D fan-out. The fan-in and fan-out insertion losses for each core are measured to be $4.4 \mathrm{~dB}$. In this case, $150 \mathrm{~m}$ of MCF are used to demonstrate the connectivity concept between a RRH and the antenna at the top of a building. However, as demonstrated in previous works, for the same optical power level injected to the fibre, we obtained a minimum of $5 \mathrm{~dB}$ power margin at the receiver to meet the specifications of the different multi-RAT services [17]. Thus, considering the MCF attenuation of $0.45 \mathrm{~dB} / \mathrm{km}$, with $5 \mathrm{~dB}$ power margin the fibre reach could be extended to several $\mathrm{km}$.

At the reception, direct detection is employed with PIN photodetectors. Variable optical attenuators (VOAs) are employed before photodetection in order to evaluate the RoMCF transmission performance for different received signal to noise ratio (SNR) levels, using the same power level injected into the fibre and with the same crosstalk levels in the MCF for all the services evaluated.

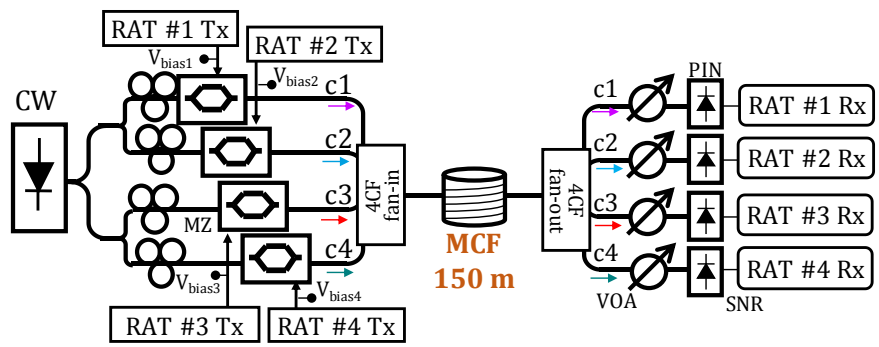

Fig. 2. Experimental setup for multi-RATs provision using RoMCF fronthaul 


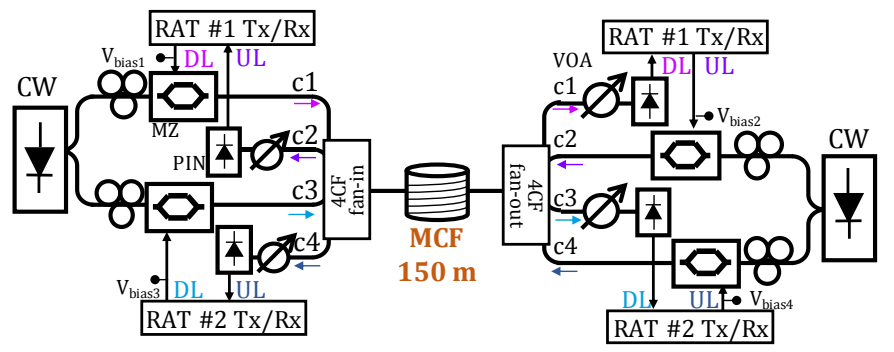

Fig. 3. Experimental setup for bi-directional multi-RATs provision using RoMCF fronthaul

Once received, the quality of the cellular signals is evaluated with the wireless test set and analysed with Vector Signal Analyser 89600 software (revision 20.2). The received quality of each signal (considering GSM, EDGE, EGPRS2-A, cdma2000 1xEV-DO, UMTS HSPA+ and LTE-Advanced) is compared with the 3GPP standard requirements at the antenna, summarized in Table 1 . The minimum SNR level needed to meet the standards specification after RoMCF transmission is obtained for each case.

Table 1. Antenna Requirements for each Cellular Standard

\begin{tabular}{|l|l|}
\hline Cellular Standard & Antenna Requirement \\
\hline 3GPP GSM GMSK & $\begin{array}{l}\text { RMS phase error }<5^{\circ} \\
\text { Phase error peak }<20^{\circ}\end{array}$ \\
\hline 3GPP GSM 8PSK & RMS EVM $<7 \%$ \\
\hline 3GPP EGPRS2-A & $\begin{array}{l}\text { RMS EVM }<5 \% \\
(16 Q A M \text { or } 32 Q A M)\end{array}$ \\
\hline 3GPP2 cdma2000 1xEV-D0 & Rho $>0.97$ \\
\hline 3GPP UMTS HSPA+ & RMS EVMQPSK $<17.5 \%$ \\
\hline 3GPP LTE-A & RMS EVM 16 QAM $<12.5 \%$ \\
\hline
\end{tabular}

As depicted in Table 1, the most common methods for measuring the quality of a cellular signal are with the error vector magnitude (EVM) and rho parameter. Both EVM and rho measure the likeness of the actual signal compared to the ideal reference signal. The EVM is defined as a percentage error, where the error vector represents the vector distance between the phase and magnitude of a recovered symbol and its ideal symbol location. Thus, a perfect signal would have an EVM of $0 \%$. Rho calculates the correlation, so it has an opposite scale and it is expressed as a decimal value between 0 and 1 . In this case, a perfect signal would have a rho value of 1 . Table 1 reports the most restrictive antenna requirements defined by each standard in terms of phase error, EVM or rho. In the next section, the characteristics of each cellular service are further described and the requirements after multicore fibre transmission are analysed experimentally.

\section{Multi-RATs Performance over Multicore Fibre}

\subsection{RAT\#1: GSM performance in RoMCF}

In the experimental study of RoMCF transmission of 2G RATs, the performance of GSM is evaluated employing the experimental setup depicted in Fig. 2. The GSM performance is evaluated with the generation of a mixed signal comprising GSM, EDGE and EGPRS2-A (with 16QAM and 32QAM modulation) with 13 frames in the same carrier and normal timeslots generated with a symbol rate of $270.833 \mathrm{ksps}$. The global system for mobile communications (GSM) was first defined to use Gaussian minimum shift keying (GMSK). As depicted in Table 1 antenna requirements, according to 3GPP standards for a single repeater, the phase error in GSM using GMSK modulation shall not be greater than $5^{\circ}$ with a maximum peak deviation of $20^{\circ}$ degrees [19]. 
In the evolution of GSM to EDGE technology, the system employs 3/8 $\pi$ 8PSK modulation and, according to $3 \mathrm{GPP}$ technical specification, it requires a root mean squared (RMS) EVM smaller than $7 \%$ [19]. Next, in the evolution to EGPRS2-A signals with 16 and 32QAM modulation, the 3GPP definition for a base transceiver station (BTS) in normal condition (most restrictive) states that the EVM $_{\text {RMS }}$ of the output RF signal shall not exceed 5\% [19]. This mixed signal for 2G and 2.5G services is generated according to E-GSM900 band with a centre frequency of $930 \mathrm{MHz}$ and is transmitted over core $\mathrm{c} 1$ following the experimental setup depicted in Fig. 2.

Fig. 4 shows the experimental results obtained for 2G GSM service using GMSK modulation. It can

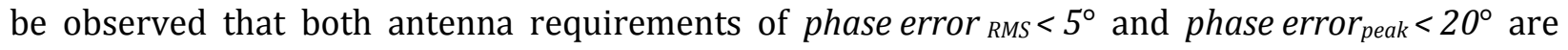
fulfilled for received SNR values higher than $25 \mathrm{~dB}$.
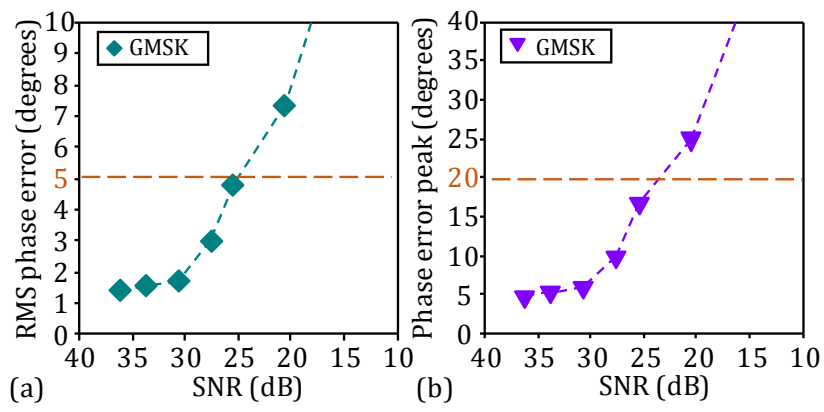

Fig. 4. (a) Experimental RMS phase error and (b) phase error peak of RAT\#1 GSM employing GMSK vs. received SNR after RoMCF transmission over core $\mathrm{c} 1$ of Fig. 2. Standard requirement represented as horizontal dashed lines

Fig. 5 shows the measured GMSK reference time and data constellations for a received $\mathrm{SNR}=25 \mathrm{~dB}$, confirming the correct operation of this RAT after multicore fibre transmission in coexistence with the other RATs evaluated in this work. The obtained requirements of GSM employing GMSK are summarized in Table 2.

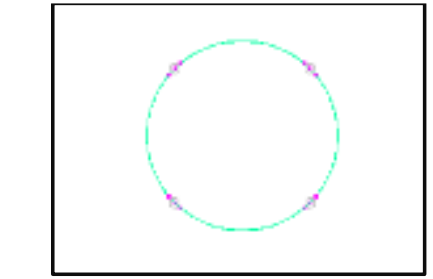

(a)
GMSK reference time

(b)

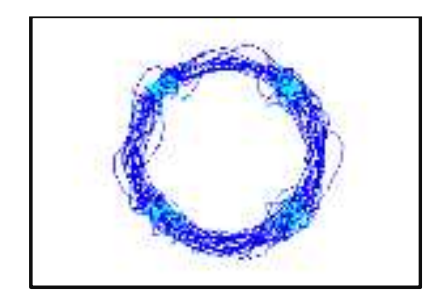

$\mathrm{EVM}=12.37 \%$

Fig. 5. Measured GMSK (a) reference time and (b) data constellation for RAT\#1 GSM with received SNR=25 dB after RoMCF fibre transmission over core c1 of Fig. 2.

Table 2. 3GPP GSM GMSK Specifications Summary

\begin{tabular}{|l|l|}
\hline Main characteristics & Antenna Requirement \\
\hline GMSK modulation & RMS phase error $<5^{\circ}$ \\
Channel spacing $=200 \mathrm{kHz}$ & Phase error peak $<20^{\circ}$ \\
Symbol rate $=270.833 \mathrm{ksps}$ & RoMCF SNR $>25 \mathrm{~dB}$ \\
\hline
\end{tabular}

Next, in Fig. 6 the experimental results obtained for 2G GSM service employing EDGE with 8PSK modulation are represented. In this case, the antenna requirements are met also for SNR values higher than $25 \mathrm{~dB}$. It can be observed in the constellations included in Fig. 6(b) that for lower SNR values, e.g. SNR=20 dB, the received EVM is above the $3 \mathrm{GPP}$ recommendation of $7 \%$. 

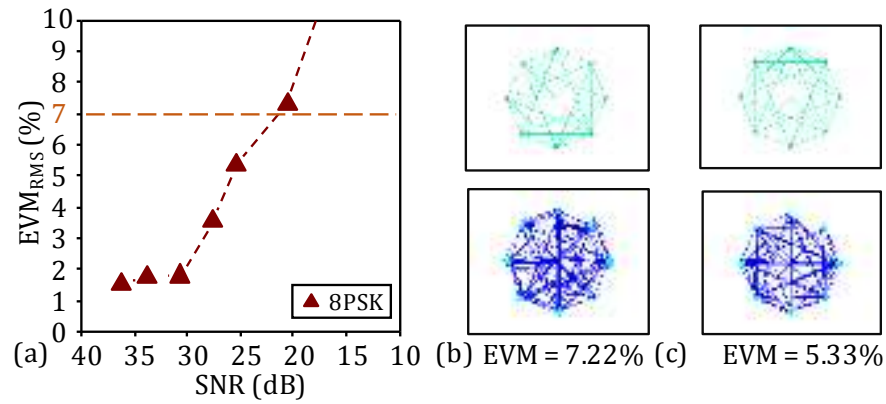

(b) $\mathrm{EVM}=7.22 \%(\mathrm{c})$

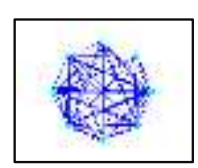

$\mathrm{EVM}=5.33 \%$

Fig. 6. (a) Experimental EVM RMs for RAT\#1 GSM employing EDGE with 8PSK modulation vs. received SNR after RoMCF transmission over core c1 of Fig. 2. Measured reference signals and data constellations for (b) SNR=20 dB and

(c) $\mathrm{SNR}=25 \mathrm{~dB}$

Table 3. 3GPP GSM EDGE with 8PSK Specifications Summary

\begin{tabular}{|l|l|}
\hline Main characteristics & Antenna Requirement \\
\hline $3 / 8 \pi$ 8PSK modulation & RMS EVM $<7 \%$ \\
Channel spacing $=200 \mathrm{kHz}$ & RoMCF SNR $>25 \mathrm{~dB}$ \\
Symbol rate $=270.833 \mathrm{ksps}$ & \\
\hline
\end{tabular}

The experimental results for GSM RAT employing EGPRS2-A with both 16QAM and 32QAM modulations are represented in Fig. 7. The 3GPP requirement of EVM below 5\% is met also for received SNR values higher than $25 \mathrm{~dB}$. Examples of received reference signals and data constellations are included in Fig. 7(b-c) for both modulation orders.
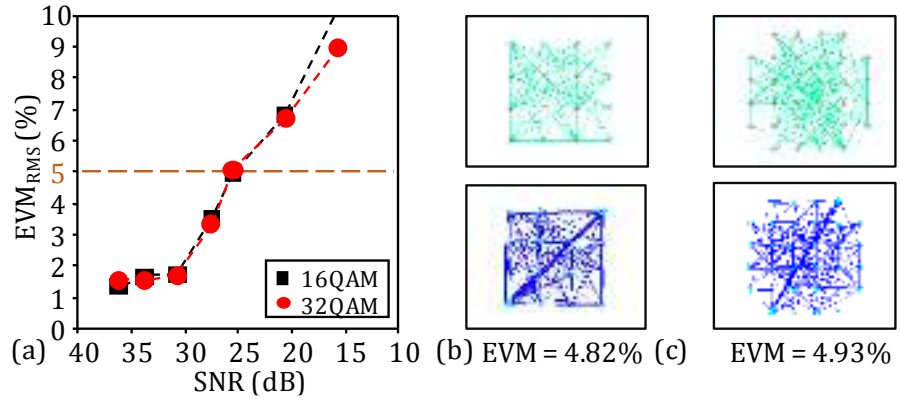

(b) $\mathrm{EVM}=4.82 \%$ (c)

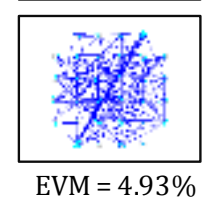

Fig. 7. (a) Experimental EVMrms for RAT\#1 GSM employing EGPRS2-A with 16QAM and 32QAM modulation vs. received SNR and measured reference signals and data constellations for SNR=25 dB for (b) 16QAM and (c) 32QAM modulation after RoMCF over core c1 of Fig. 2

Table 4. 3GPP GSM EGPRS2-A Specifications Summary

\begin{tabular}{|l|l|}
\hline Main characteristics & Antenna Requirement \\
\hline $\begin{array}{l}\text { 16QAM or 32QAM modulation } \\
\text { Channel spacing }=200 \mathrm{kHz} \\
\text { Symbol rate } 270.833 \mathrm{ksps}\end{array}$ & RMS EVM $<5 \%$ \\
\hline
\end{tabular}

\subsection{RAT\#2: UMTS HSPA+ performance in RoMCF}

The evolution of the universal mobile telecommunications system (UMTS) standard is based on high-speed packet access, also known as evolved HSPA or HSPA+. 3GPP defines that for HSPA+ signals employing QPSK modulation, the EVM should not exceed 17.5\% [20]. For the evaluation of 3.9G services provision using RoMCF, a HSPA+ signal comprising a $10 \mathrm{~ms}$ frame in a $5 \mathrm{MHz}$ carrier is generated at a centre frequency of $2.14 \mathrm{GHz}$ and is transmitted over core c2 following the experimental setup depicted in Fig. 2. Fig. 8 shows the experimental results in terms of EVM $\mathrm{RMS}_{\mathrm{R}}$ and rho for different received SNR levels. 

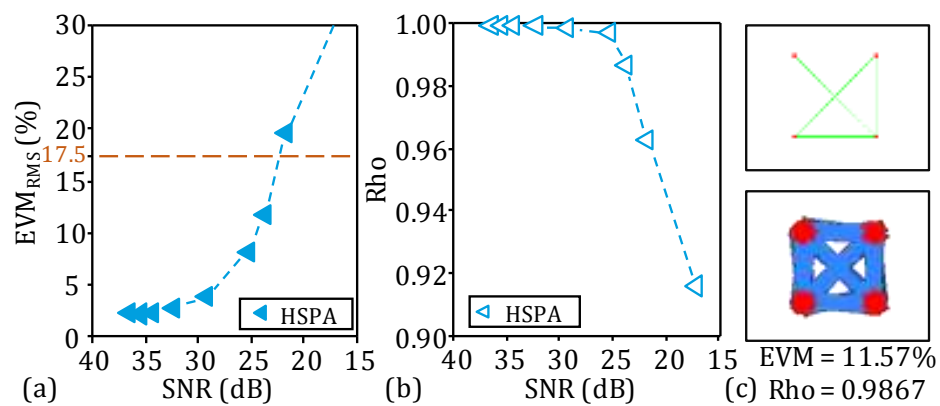

Fig. 8. (a) Experimental EVMRMS and (b) measured rho for RAT\#2 HPSA+ vs. received SNR after RoMCF over core c2 of Fig. 2 and (c) measured reference signals and data constellations for SNR=23.7 dB

Table 5. 3GPP HSPA+ Specifications Summary

\begin{tabular}{|l|l|}
\hline Main characteristics & Antenna Requirement \\
\hline QPSK or 16QAM & \\
modulation & RMS EVM \\
$5 \mathrm{MHSK}<17.5 \%$ \\
Chip rate $=3.84 \mathrm{Mcps}$ & RoMCF SNR $>23 \mathrm{~dB}$ \\
\hline
\end{tabular}

The EVM requirement at the antenna is meet for SNRs higher than $23 \mathrm{~dB}$, where the rho value is better than 0.98 , as confirmed by the received constellation included in Fig. 8(c). The specification summary for the correct RoMCF transmission of HSPA+ is included in Table 5.

\subsection{RAT\#3: cdma2000 1xEV-DO performance in RoMCF}

In the evolution of cellular signals, the 3GPP2 standards committee defined cdma2000 $1 \mathrm{x}$ Evolution Data Only (1xEV-D0) to increase up to ten times the rate of the original IS-95 systems. The reverse link (from the mobile back to the BTS) is enhanced to support higher complexity modulation. For this reason, $3.5 \mathrm{G}$ cdma2 000 1xEV-DO signals are evaluated with the bi-directional setup depicted in Fig. 3 employing a centre frequency $\mathrm{f}_{c}=942.5 \mathrm{MHz}$ for the forward carriers and $\mathrm{f}_{\mathrm{c}}=897.5 \mathrm{MHz}$ for the reverse carriers. In this demonstration, carrier aggregation is also included by generating two cdma2000 1xEV-D0 carriers separated $\Delta \mathrm{f}= \pm 1.25 \mathrm{MHz}$. For simplicity, the reported EVM values in this section correspond to CC1 located at $\mathrm{f}_{\mathrm{c}}+1.25 \mathrm{MHz}$.

Following the bi-directional setup depicted in Fig. 3 the forward carriers are transmitted over core $\mathrm{c} 1$ and the reverse carriers are transmitted in the opposite direction over core $\mathrm{c} 2$. In the requirements of $1 \mathrm{xEV}$-DO systems, the overall rho at the BTS must be greater than 0.912 [21] and it is recommended to be higher than 0.97 for a good link [21][22].
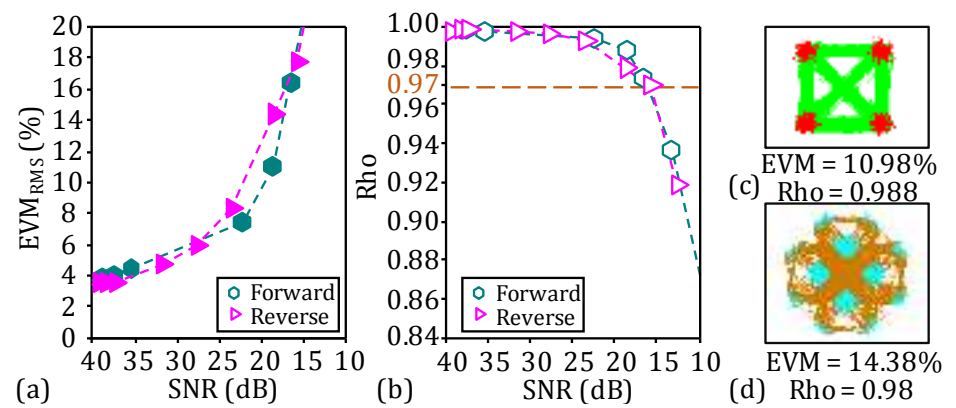

Fig. 9. (a) Experimental EVMRMs and (b) measured rho for RAT\#3 1xEV-DO vs. received SNR after RoMCF over core c1 and c2 in Fig. 3 and measured constellations with SNR=18.5 dB for (c) forward and (d) reverse signals

Table 6. 3GPP 1xEV-DO Specifications Summary

\begin{tabular}{|l|l|}
\hline Main characteristics & Antenna Requirement \\
\hline $1.25 \mathrm{MHz}$ bandwidth & Rho $>0.97$ \\
$\begin{array}{l}\text { Chip rate }=1.2288 \mathrm{Mcps} \\
\text { Pilot PN sequence period }=26.666 \mathrm{~ms} \\
\text { Carrier aggregation compatible }\end{array}$ & RoMCF SNR $>18.5 \mathrm{~dB}$ \\
\hline
\end{tabular}


Fig. 9(a-b) shows the measured EVM and rho for 1xEV-DO RAT considering both forward and reverse carriers. The RoMCF transmission in both directions meets the 3GPP rho requirement for SNR higher than $18.5 \mathrm{~dB}$ as confirmed by the constellations included in Fig. 9(c-d). The requirements obtained for 3GPP 1xEV-DO RAT are included in Table 6.

\subsection{RAT\#4: LTE-A performance in RoMCF}

LTE-A is a $4 \mathrm{G}$ mobile communication standard [23] meeting the requirements of the IMTAdvanced standard. In this case, for 4G RAT, we evaluate the RoMCF performance of both LTE-A downlink (DL) and uplink (UL) signals. Fully standard 3GPP LTE-A carrier-aggregated signals are generated at the centre of cellular FDD band 7: LTE-A DL carriers are centred at $\mathrm{f}_{\mathrm{c}}=2.655 \mathrm{GHz}$ and LTE-A UL carriers at $\mathrm{f}_{\mathrm{c}}=2.535 \mathrm{GHz}$. The experimental evaluation is performed including also carrier aggregation comprising two $20 \mathrm{MHz}$ component carriers (CCs) separated $\Delta \mathrm{f}= \pm 9.9 \mathrm{MHz}$. The RoMCF performance with carrier separation was evaluated in previous works confirming the correct operation of aggregated carriers if they are not overlapped in spectrum [24].

Following the bi-directional setup depicted in Fig. 3, two carrier-aggregated DL $20 \mathrm{MHz}$ LTE-A single carriers (SISO) are transmitted over core c3; and two UL 20 MHz LTE-A carriers over core c4. For simplicity, the reported EVM values of this section correspond to CC1 located at $\mathrm{f}_{\mathrm{c}}+9.9 \mathrm{MHz}$ (worst case). Fig. 10 reports the measured EVM and constellations for LTE-A SISO signals in DL and in UL transmission. Observing the constellations, we can observe the difference between DL and UL, in particular the physical uplink shared channel (PUSCH) demodulation reference signal with the Zadoff-Chu sequence, which results in constellation points on a circle.

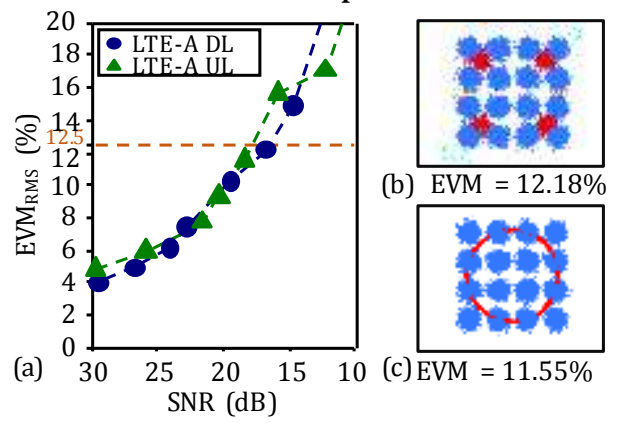

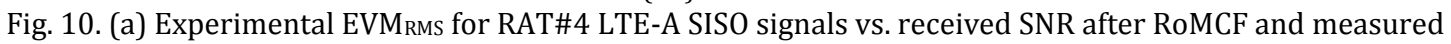
constellations for (b) DL over core c3 of Fig. 3 for SNR=16.7 dB and (c) UL SISO signals over core c4 of Fig. 3 for $\mathrm{SNR}=18.3 \mathrm{~dB}$

Current LTE-A cellular communication standard also implements multi-antenna multiple-input multiple-output (MIMO) multiplexing. MIMO is used to increase the overall bitrate through the transmission of two or more different data streams on different antennas [18]. In this case, the performance of MIMO LTE-A signals is evaluated employing the setup depicted in Fig. 2 as MIMO spatial multiplexing is only implemented in the downlink. $2 \times 2$ MIMO spatial multiplexing is implemented over cores c3 and c4 of Fig. 2. According to 3GPP LTE-A implementation, the cellspecific reference signal (C-RS) in $2 \times 2$ MIMO implement a frequency multiplexing between antenna port 0 and 1 as depicted in Fig. 11(a). The downlink reference signals are known-symbols inserted into the downlink time-frequency grid used for channel quality estimation for channel state information reporting and for mobility measurements. According to this channel estimation, the user equipment (UE) sends a message to the eNB (evolved NodeB) scheduler with the codebook index. The precoding choices are defined in a lookup table as included in the example of Table 7 for spatial multiplexing over 2 layers. In this evaluation, index 2 is used for MIMO processing. In addition, when generating the MIMO multiplexed signals, one port is called master and the others are called slaves. At reception, the master signal must be present in order to properly synchronize with the slave signals. As it is depicted in the example of Fig. 11(b), when both the master (signal in core c3) and 
the slave (signal at core c4) are received, the $2 \times 2$ data can be demodulated, analysed and its EVM is reported. If the slave signal is not found, as depicted in Fig. 11(c), only the synchronization from the master is observed and the data channels cannot be demodulated. It can be clearly seen that in this case, although the transmitted data signals are mapped with 16QAM, the represented constellation in Fig. 11(c) is mapped in QPSK plus the pilots corresponding to the LTE synchronization. Next, as shown in Fig. 11(d), if the master is not present, no signal can be recovered.

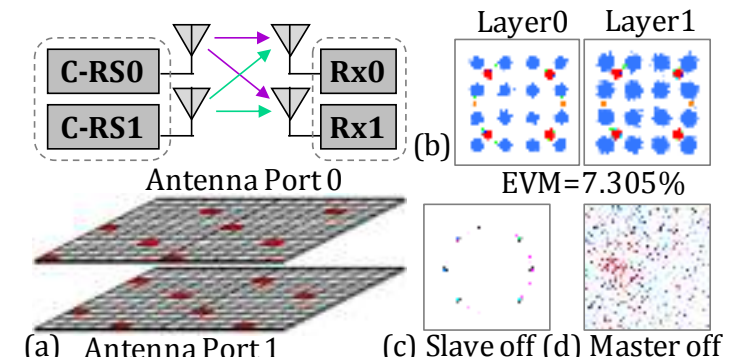

Fig. 11. (a) LTE-A CS-RS grid per antenna ports in MIMO $2 \times 2$ configuration. Operation example when: (b) both channels are present (correct reception example for SNR of $28 \mathrm{~dB}$ ), (c) the slave signal is not present, and (d) the master signal is not found

Table 7. 3GPP LTE codebook index for $2 \times 2$ MIMO implementation over 2 layers

\begin{tabular}{|c|c|}
\hline Codebook index & 2 layers \\
\hline 0 & $\frac{1}{\sqrt{2}}\left[\begin{array}{ll}1 & 0 \\
0 & 1\end{array}\right]$ \\
\hline 1 & $\frac{1}{2}\left[\begin{array}{cc}1 & 1 \\
1 & -1\end{array}\right]$ \\
\hline 2 & $\frac{1}{2}\left[\begin{array}{cc}1 & 1 \\
j & -j\end{array}\right]$ \\
\hline
\end{tabular}

¡Error! No se encuentra el origen de la referencia.Fig. 12(a) shows the performance of CC1 of two-aggregated 4G LTE-A $20 \mathrm{MHz}$ carriers implementing $2 \times 2 \mathrm{MIMO}$ spatial multiplexing. The received constellation examples for both layers with $S N R=28 \mathrm{~dB}$ is included in Fig. 11(a). In order to analyse in more detail the performance of each channel, the EVM of the physical downlink shared channel (PDSCH)[25] in each layer using 16QAM is measured and reported in Fig. 12(b).
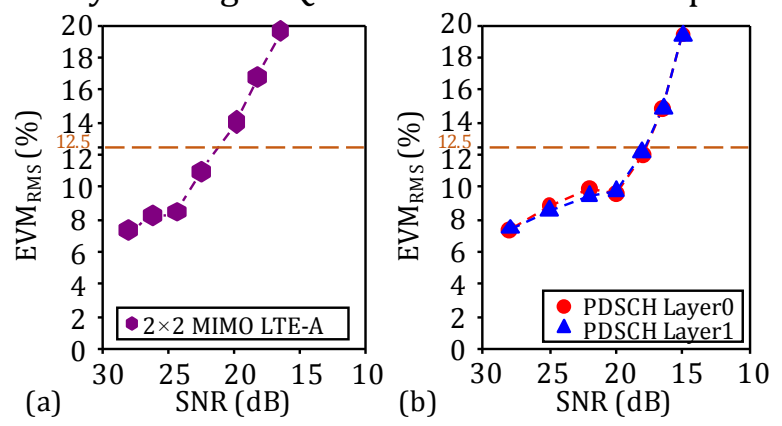

Fig. 12. (a) Experimental EVMrms for RAT\#4 LTE-A implementing 2×2 MIMO multiplexing over cores c3 and c4 in Fig. 2.

(b) Measured EVM for data channel PDSCH 16QAM of each layer in $2 \times 2$ configuration

Comparing Fig. 10(a) with Fig. 12(a), it can be observed that $2 \times 2$ MIMO requires higher SNR in order to provide double bitrate over the same bandwidth. In this case, $2 \times 2$ MIMO LTE-A meets the EVM requirement at the antenna for an SNR in both cores higher than $20.2 \mathrm{~dB}$.

In order to further expand the capacity and increasing the number of antennas, the performance of $4 \times 4$ MIMO LTE-A signals was evaluated with spatial multiplexing over the four cores of the fronthaul system depicted in Fig. 2. In this case, the data is multiplexed in 4 layers, each one performing as depicted in Fig. 13(b). 

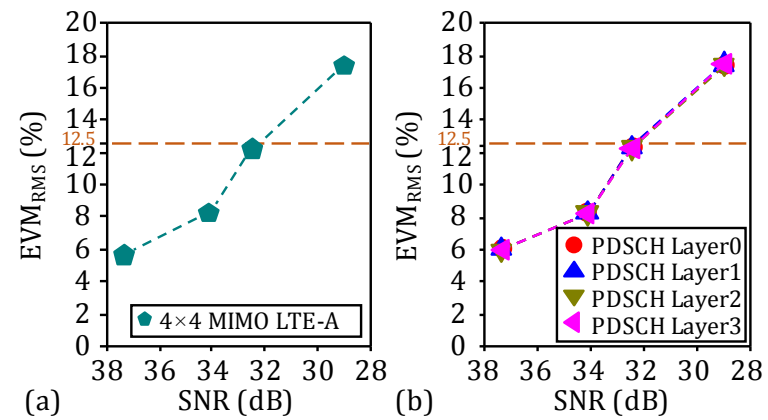

Fig. 13. (a) Experimental EVMRMS for RAT\#4 LTE-A 4×4 MIMO signals multiplexing over four cores in Fig. 2 vs. received SNR after RoMCF. (b) Measured EVM for data channel PDSCH 16QAM of each layer in $4 \times 4$ configuration

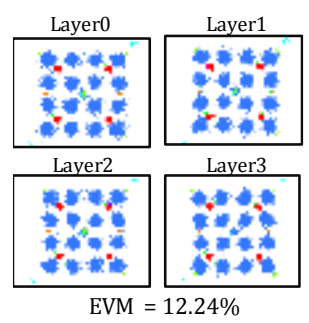

Fig. 14. Measured constellation and EVM for a $4 \times 4$ MIMO signal implementing spatial multiplexing with a SNR=32.4 $\mathrm{dB}$

The experimental results reported in Fig. 13 point out that a 4-antenna system requires a received SNR higher than $32.4 \mathrm{~dB}$ while increasing $\times 4$ the bitrate provided to the user. Fig. 13 shows a constellation example for the four layers with a SNR of $32.4 \mathrm{~dB}$. To conclude with this experimental evaluation, Table 8 summarizes the SNR requirements for the different configurations of LTE-A signals.

Table 8. 3GPP LTE-A Specifications Summary

\begin{tabular}{|l|l|}
\hline Main characteristics & Antenna Requirement \\
\hline OFDM modulation & RMS EVM $_{16 \mathrm{QAM}}<12.5 \%$ \\
$10 \mathrm{~ms}$ frame & RoMCF SNR $_{\mathrm{DL}}>16.7 \mathrm{~dB}$ \\
Up to $20 \mathrm{MHz}$ channel bandwidth & RoMCF SNR $_{\mathrm{UL}}>18.3 \mathrm{~dB}$ \\
Carrier aggregation compatible & RoMCF SNR $_{2 \times 2}$ MIMO $>20.2 \mathrm{~dB}$ \\
& RoMCF SNR $_{4 \times 4} \mathrm{MIMO}>32.4 \mathrm{~dB}$ \\
\hline
\end{tabular}

\section{Conclusion}

In this paper we evaluated experimentally a next-generation optical fronthaul employing spacedivision multiplexing in radio-over-multicore-fibre. One of the requirements of next-generation networks is to flexibly integrate the already deployed heterogeneous multi-radio access technologies ranging from $2 \mathrm{G}$ to $4 \mathrm{G}$. The proposed RoMCF fronthaul supports multi-RATs simultaneous provision and advanced MIMO capabilities to ensure cellular standard compatibility with next-generation 5G networks. In this experimental evaluation we analysed the multiplexed transmission of 2G, 3G, 3.9G and 4G MIMO signals using RoMCF transmission over a commercially available four-core MCF. The signal-to-noise ratio required at the receiver is evaluated for each radio access technology (RAT). 2G RAT evaluation comprises GSM, EDGE and EGPRS2-A signals RoMCF performance. The experimental study also includes bi-directional signal transmission of 3.5G cdma2000 including forward and reverse 1xEV-DO signals. UMTS HSPA+ 3.9G RAT was also evaluated. Bi-directional 4G LTE-A RATs were also studied in both directions with DL and UL carriers. In addition, the experimental 4G evaluation includes also multi-antenna transmission with $2 \times 2$ and $4 \times 4$ MIMO spatial multiplexing over two and four cores in the MCF, respectively. 
Taking into account the antenna quality requirements in terms of EVM, phase error or rho specified by each cellular standard, the minimum SNR required after RoMCF was obtained experimentally. Table 9 summarizes the SNR requirements for each cellular signal.

\begin{tabular}{|c|c|}
\hline Cellular Standard & $\begin{array}{l}\text { RoMCF SNR } \\
\text { Requirement }\end{array}$ \\
\hline $\begin{array}{l}\text { 3GPP GSM GMSK } \\
\text { 3GPP GSM 8PSK } \\
\text { 3GPP EGPRS2-A } \\
\end{array}$ & $\mathrm{SNR}>25 \mathrm{~dB}$ \\
\hline 3GPP UMTS HSPA+ & $\mathrm{SNR}>23 \mathrm{~dB}$ \\
\hline 3GPP2 cdma2000 1xEV-DO & $\mathrm{SNR}>18.5 \mathrm{~dB}$ \\
\hline 3GPP LTE-A & $\begin{array}{l}\mathrm{SNR}_{\mathrm{DL}}>16.7 \mathrm{~dB} \\
\mathrm{SNR}_{\mathrm{UL}}>18.3 \mathrm{~dB} \\
\mathrm{SNR}_{2 \times 2} \text { MIMO }>20.2 \mathrm{~dB} \\
\mathrm{SNR}_{4 \times 4} \text { MIMO }>32.4 \mathrm{~dB}\end{array}$ \\
\hline
\end{tabular}

The proposed multicore-fibre optical fronthaul also enables the implementation of spatial multiplexed MIMO over different cores, transmitting different data streams over each core and thus, increasing the provided data rate to the user with multiple antennas. The experimental results indicate that single-antenna and two-antenna systems implementing $2 \times 2$ MIMO multiplexing over two cores meet the antenna requirements for received SNR values higher than $25 \mathrm{~dB}$. Employing a higher number of antennas would require higher SNR if MIMO spatial multiplexing is implemented to increase the provided data rate. Antenna diversity transmitting the same data through different antennas does not require extra SNR but does not provide higher data rate either. In the case of $4 \times 4$ MIMO multiplexing over four cores of MCF fronthaul transmission, $32 \mathrm{~dB}$ SNR is needed to achieve almost four times the provided bitrate per user. These dimensioning SNR values provide the basis guidelines for next-generation mobile access networks comprising multicore-fibre optical fronthaul and ensuring multi-RATs coexistence.

\section{Acknowledgments}

This research was supported in part by Spain National Plan MINECO/FEDER UE TEC2015-70858-C21-R XCORE and GVA AICO/2018/324 NXTIC projects. The work of M. Morant is supported by Spain Juan de la Cierva IJCI-2016-27578 grant and Fundacion BBVA Leonardo 2018 HYPERCONN project.

\section{References}

[1] C. Ranaweera, E. Wong, A. Nirmalathas, C. Jayasundara and C. Lim, "5G C-RAN with Optical Fronthaul: An analysis from a deployment perspective," IEEE/OSA Journal of Lightwave Technology, vol. 36, no. 11, pp. 2059-2068, June 2018.

[2] M. Patzold, "5G Readiness on the Horizon [Mobile Radio]," IEEE Vehicular Technology Magazine, vol. 13, no. 1, pp. 6-13, March 2018.

[3] J. Zeng, Y. Cao, F. Ke, M. Huang, G. Zhang and W. Lu, "Performance evaluation of secure multipath retransmission mechanism in next generation heterogeneous communication systems," IET Networks, vol. 7, no. 2, pp. 61-67, March 2018.

[4] T. Nakamura, A. Benjebbour, Y. Kishiyama, S. Suyama, T. Imai, "5G Radio Access: Requirements, concept and experimental trials," IEICE Transactions on Communications , vol. E98-B, no. 8, pp. 13971406, Aug. 2015

[5] S.-Y. Lien, S.-L. Shieh, Y. Huang, B. Su, Y-L. Hsu and H-Y. Wei, "5G New Radio: Waveform, frame structure, multiple access, and initial access," IEEE Communications Magazine, vol. 55, no. 6, pp. 64-71, June 2017. 
[6] I. A. Alimi, A. L. Teixeira and P. P. Monteiro, "Towards an efficient C-RAN optical fronthaul for the future networks: A tutorial on technologies, requirements, challenges, and solutions," IEEE Communications Surveys \& Tutorials, vol. 20, no. 1, pp. 708-769, 2018.

[7] B. G. Kim, S. H. Bae, H. Kim and Y. C. Chung, "Feasibility of RoF-based optical fronthaul network for next-generation mobile communications," In Proc. Opto-Electronics and Communications Conference (OECC) and Photonics Global Conference (PGC), Nov. 2017

[8] Z. Gao, L. Dai, D. Mi, Z. Wang, M. A. Imran, and M. Z. Shakir, "MmWave massive-MIMO-based wireless backhaul for the 5G ultradense network," IEEE Wireless Communications, vol. 22, no. 5, pp. 13-21, Oct. 2015.

[9] D. Wake, A. Nkansah, and N. J. Gomes, "Radio over fiber link design for next generation wireless systems," IEEE/OSA Journal of Lightwave Technology, vol. 28, no. 16, pp. 2456-2464, Aug. 2010.

[10] A. Haddad and M. Gagnaire, "Radio-over-Fiber (RoF) for mobile backhauling: A technical and economic comparison between analog and digitized RoF," in Proc. International Conference on Optical Network Design and Modeling (ONDM), pp. 132-137, May 2014.

[11] P. J. Urban, G. C. Amaral, and J. P. von der Weid, "Fiber monitoring using a sub-carrier band in a subcarrier multiplexed radio-over-fiber transmission system for applications in analog mobile fronthaul," IEEE/OSA Journal of Lightwave Technology, vol. 34, no. 13, pp. 3118-3125, July 2016.

[12] K. Saitoh and S. Matsuo, "Multicore fiber technology" IEEE/OSA Journal of Lightwave Technology, vol. 34, no. 1, pp.55-66, January 2016.

[13] S. Inao, T. Sato, S. Sentsui, T. Kuroha, and Y. Nishimura, "Multicore optical fiber," in Proc. Optical Fiber Communication Conference, paper WB1, March 1979.

[14] R. J. Essiambre, G. Kramer, P. J. Winzer, G. J. Foschini, and B. Goebel, "Capacity limits of optical fiber networks," IEEE/OSA Journal of Lightwave Technology, vol. 28, no. 4, pp. 662-701, Feb. 2010.

[15] K. Nakajima, T. Matsui, K. Saito, T. Sakamoto, N. Araki, "Multi-Core Fiber Technology: Next Generation Optical Communication Strategy," IEEE Communications Standards Magazine, vol. 1, no. 3, pp. 38-45, Oct. 2017.

[16] R. Wang, H. Hu and X. Yang, "Potentials and challenges of C-RAN Supporting multi-RATs toward 5G mobile networks,", IEEE Access, vol. 2, pp. 1187-1195, Oct. 2014

[17] M. Morant and R. Llorente, "Reconfigurable radio-over-multicore optical fronthaul for seamless 2G, UMTS and LTE-A MIMO wireless provision," in Proc. Optical Fiber Communications Conference and Exhibition (OFC), p. W2A.43, March 2017.

[18] M. Morant and R. Llorente, "5G large-count MIMO fronthaul systems based on multicore microwave photonics," in Proc. International Topical Meeting on Microwave Photonics (MWP), Oct. 2017.

[19] 3GPP TS 45.005 V8.8.0 Technical Specification, "3rd Generation Partnership Project; Technical Specification Group GSM/EDGE Radio Access Network; Radio transmission and reception (Release 8)," March 2010.

[20] ETSI TS 134 121-1 V10.5.0 Technical Specification, "Universal Mobile Telecommunications System (UMTS); User Equipment (UE) conformance specification; Radio transmission and reception (FDD); Part 1: Conformance specification (3GPP TS 34.121-1 version 10.5.0 Release 10)," Feb. 2013.

[21] Agilent Technologies, "Forward Link Measurements for 1xEV-DO Access Networks," Application Note 1398, Nov. 2002.

[22] Anritsu, "Practical Tips on cdma2000 1xEV-DO Forward-Link Movement," Application Note No. 11410-00379, Rev. A, March 2007.

[23] ETSI TS 136101 V10.3.0 Technical Specification, "LTE; Evolved Universal Terrestrial Radio Access (E-UTRA); User Equipment (UE) radio transmission and reception (3GPP TS 36.101 version 10.3.0 Release 10)," June 2011.

[24] M. Morant and R. Llorente, "Performance analysis of carrier-aggregated multi-antenna 4×4 MIMO LTE-A fronthaul by spatial multiplexing on multicore fiber," IEEE/OSA Journal of Lightwave Technology, vol. 36, no. 2, pp. 594-600, Jan. 2018.

[25] J. Zhu and H. Li, "On the performance of LTE Physical Downlink Shared Channel", Proceedings of in Proc. International Conference on Computer Science and Network Technology, vol. 2, pp. 983-986, Dec. 2011. 